\title{
Leserterapia como adjuvante na reparação de lesões químicas em boca
}

\author{
Lasertherapy as an adjuvante in the repair of Chemical lesions in the mouth \\ La terapia con láser com o coadyuvante em la reparación de lesiones químicas em la boca
}

Recebido: 26/10/2021 | Revisado: 05/11/2021 | Aceito: 11/11/2021 | Publicado: 14/11/2021

\author{
Regina Maria Raffaele \\ ORCID: https://orcid.org/0000-0002-1636-3688 \\ Associação Beneficente Santa Casa de Campo Grande, Brasil \\ E-mail: reginaraffaele@hotmail.com \\ Mario Eduardo Baldo \\ ORCID: https://orcid.org/0000-0003-0026-595X \\ Associação Beneficente Santa Casa de Campo Grande, Brasil \\ E-mail: m.e.baldo@gmail.com \\ Yara Diniz Souza \\ ORCID: https://orcid.org/0000-0001-7845-0130 \\ Hospital Universitário Maria Aparecida Pedrossian, Brasil \\ E-mail: dinizsouzay@gmail.com \\ Ellen Cristina Gaetti-Jardim \\ ORCID: https://orcid.org/0000-0003-2471-465X \\ Hospital Universitário Maria Aparecida Pedrossian, Brasil \\ E-mail: ellen.jardim@ufms.br
}

\begin{abstract}
Resumo
O objetivo deste trabalho é relatar e discutir o caso de um paciente de 38 anos de idade, melanodermo, sexo masculino, internado na Associação Beneficente Santa Casa de Campo Grande, por tentativa de suicídio por ingesta de soda caustica, causando inúmeras ulceras em todo sistema gastrointestinal, em especial a cavidade oral apresentase com edema de grande extensão, mucosas friáveis e ulceradas, assim para melhora e regeneração mais rápida foi usado terapia fotodinâmica e terapia de fotobiomodulação com o uso do laser de baixa potência. No transcorrer da internação, o paciente apresentou melhora significativa da cicatrização de mucosas orais e língua, evidenciando uma forma de tratamento não invasiva e com ótima resposta terapêutica.
\end{abstract}

Palavras-chave: Terapia com luz de baixa intensidade; Queimaduras químicas; Tentativa de suicídio.

\begin{abstract}
The objective of this paper is to report and discuss the case of a 38-year-old, black male patient, admitted to the Santa Casa de Campo Grande Beneficent Association, for a suicide attempt by ingesting caustic soda, causing numerous ulcers throughout the system. Gastrointestinal, especially the oral cavity presents with edema of great extension, friable and ulcerated mucous membranes, so for improvement and faster regeneration, photodynamic therapy and photobiomodulation therapy eith the use of low level laser was used. During hospitalization, the patient showed significant improvement in the healing of oral mucosa and tongue, showing a non-invasive from of treatment and with an axcellent therapeutic response.
\end{abstract}

Keywords: Low intensity light therapy; Chemical burns; Suicide attempt.

\section{Resumen}

El objetivo de este trabjo es reportar y discutir el caso de un paciente varón de raza negra de 38 años, ingresado en la Asociación Benéfica Santa Casa de Campo Grande, por un intento de suicidio por ingestión de soda cáustica que le provocó numerosas úlceras en todo el sistema gastro-intestinal, especialmente la cavidad bucal se presenta con edema de gran extención, mucosas friables y ulceradas, por lo que para su mejora y una regeneración más rápida se utilizó terapia fotodinámica y terapia de fotobiomodulación con el uso de láser de baja potência. Durante la hospitalización, el paciente mostró uns mejoría significativa en la cicatrización de la mucosa oral y la lengua, mostrando una forma de tratamiento no invasiva y con una excelente respuesta terapéutica.

Palabras clave: Terapia de luz de baja intensidad; Quemaduras químicas; Intento de suicidio.

\section{Introdução}

A tentativa de suicídio pode ser caracterizada por qualquer comportamento suicida não fatal, podendo variar desde intoxicação intencional auto infligida, automutilação e demais lesões provocadas objetivando a própria morte. Em função da 
concepção mais humana do bem-estar propriamente dito, o suicídio tem se caracterizado como um problema grave de saúde pública de manejo estritamente multiprofissional (OMS, 2014). Entretanto, a literatura reporta dados escassos quanto a epidemiologia das tentativas de suicídio por ingestão de substâncias causticas, levantamentos do serviço de saúde dos Estados Unidos indicam que 2\% do total de chamadas na emergência é por ingestão de tais substâncias (Slaughter et al., 2019).

Agentes cáusticos quando em contato com pele e mucosas reagem quimicamente formando compostos ácidos, que por sua vez levam ao aparecimento de sintomas gastrointestinais como o vômito e hematêmese, cabendo a ressalva de que tanto as lesões em orofaringe quanto as gastrointestinais são caracterizadas por edema e necrose de coagulação, podendo resultar em perfuração ou perfis cicatriciais irregulares com constrição local devido a deposição de grande quantidade de fibroblastos (Vargas et al., 2016).

O tratamento proposto para a ingestão cáustica, depende da identificação do dano causado, geralmente definido pela realização de endoscopia digestiva alta nas primeiras 24 horas do acidente. Inicialmente o manejo médico deve priorizar a estabilização hemodinâmica, permeabilidade e funcionalidade das vias aéreas, a partir daí adotar o uso de inibidores da bomba de prótons. Corticosteroides, antibióticos de amplo espectro e o suporte nutricional devem ser instituídos a fim de evitar perfis inflamatórios agressivos, infecções secundárias e piora do quadro geral do paciente respectivamente (Bittencourt \& Carvalho, 2018).

A ingestão de substâncias químicas tóxicas é potencialmente danosa aos tecidos bucais, sendo a queimadura e a ulceração os resultados mais frequentes, sobretudo, em mucosa bucal e labial, podendo gerar sangramentos e obstrução de glândulas salivares, sendo esta transitória ou permanente. O tratamento é variável, de acordo com o potencial dano causado à cavidade bucal, de sintomático até cirúrgico (Varkey et al., 2006; Gilvetti et al., 2010).

Considerando que a terapia com laser de baixa potência em pacientes com queimaduras de face demonstram resultados satisfatórios em curto espaço de tempo, afetando positivamente o alívio da dor, redução de edema com subsequente melhora do epitélio e mucosas inflamadas (Raffaele et al., 2021) (Raffaele et al., 2020).

O presente relato, através da descrição de seus procedimentos e resultados obtidos visa suscitar a hipótese de que a terapia com o laser de baixa potência em conjunto com a terapia fotodinâmica podem ser empregadas com sucesso para a recuperação de pacientes que sofreram queimaduras químicas em boca restabelecendo rapidamente a integridade mucosa da orofaringe, contribuindo com a diminuição de infecções e número de dias de internação hospitalar e aumentando a qualidade de vida do paciente tratado.

\section{Metodologia e Relato de Caso}

A presente casuística trata-se de um relato com fins descritivos, exploratórios de abordagem qualitativa, com a finalidade de revelar a sua importância clínica e ser instrumento facilitador de pesquisas no que concerne o tema abordado, possibilitando a construção de revisões integrativas e possibilitando a prática baseada em evidência, a base referencial utilizada foi Souza et al., (2021) e Pereira (2018). O trabalho recebeu anuência do paciente com a assinatura do Termo de Consentimento Livre e Esclarecido e uso de direito de imagem, onde foram respeitados todos os devidos princípios éticos na condução do caso referido. Logo, este trabalho faz citação de um paciente do sexo masculino, com 38 anos de idade internado na Associação Beneficente Santa Casa de Campo Grande (Mato Grosso do Sul), após tentativa de suicídio por ingesta de um volume estimado de $500 \mathrm{ml}$ de soda caustica. Após os primeiros cuidados médicos, o paciente apresentou episódios de vômito em grande quantidade e epistaxe, progredindo com rebaixamento do nível de consciência resultando na necessidade de intubação orotraqueal. 
$\mathrm{Na}$ internação, foi realizado exame endoscópico que acusou perfuração gástrica e necrose esofágica, logo, a necessidade de cirurgia de urgência, realizada esofagogastrectomia total transhiatal, esofagostomia cervical e esplenectomia pela equipe de cirurgia geral completando no mesmo momento cirúrgico a realização de jejunostomia para nutrição.

Em relação à terapia farmacológica, o paciente fez uso de antibioticoterapia de largo espectro, analgésicos opioides, sedativos e corticosteroides.

Mediante a estabilização do quadro geral do paciente, foi realizada a avaliação odontológica, foram observadas lesões ulceradas com aspecto sanguinolento e crostoso em lábio inferior e superior, edema de grande extensão em língua e assoalho bucal, sialorreia, e lesões ulcerativas maiores em toda boca e orofaringe, como pode ser observado na figura a seguir:

Figura 1: A: Edema de grande extensão em assoalho bucal com projeção de língua; B:lesões ulceradas em mucosas labiais e dorso lingual com limitação da abertura bucal.

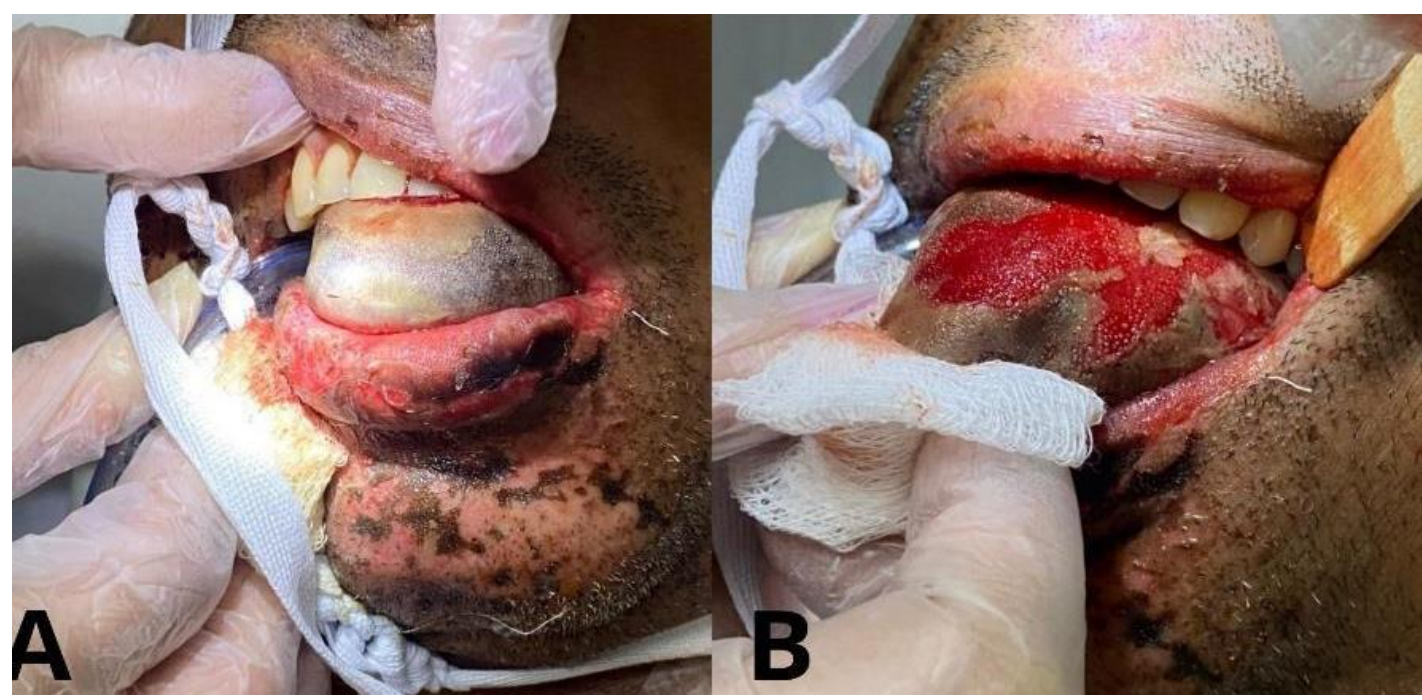

Fonte: Autores.

Definidas as condições iniciais, foi discutido juntamente com a equipe multidisciplinar e equipe médica assistente, sendo que a abordagem odontológica estabelecida foi o uso de terapia de laser de baixa potência pela equipe de odontologia hospitalar devidamente habilitada para o uso do aparelho, com a finalidade de analgesia e fomentação do processo de cicatrização da mucosa oral.

Durante a primeira e segunda sessões de PBMT (terapia de fotobiomodulação), foi utilizado o laser de baixa potência (laser Therapy EC / DMC) em contato com os tecidos. Cada área de lesão e cadeia linfática com $1 \mathrm{~cm}^{2}$ foi irradiada por 10 segundos, com $100 \mathrm{~mW}$ de potência, e $6 \mathrm{~J}$ de energia a fim de diminuir edema em lábios e língua para que conseguíssemos acessar a cavidade oral.

Em um segundo momento foi iniciado a a-PDT (terapia fotodinâmica antimicrobiana), cujo foi utilizado 0,01 \% de azul metileno aplicado nas lesões e permanecendo por 5 minutos (tempo pré-irradiação), e então foram utilizado o mesmo aparelho de laser já citado, porém com o aumento dos parâmetros energéticos para 90 segundos, ou seja, 9J por lesão.

No terceiro dia de condução da proposta terapêutica, o a a-PDT foi realizado concomitantemente com a PBMT, sendo que nos dias subsequentes apenas a PBMT foi repetida a cada 24 horas por um período de 6 dias, ajustando a carga de energia de irradiação para 3J por ponto de lesão em toda cavidade oral e região peri-labial.

Vale ressaltar que agentes tópicos adjuvantes também foram utilizados rotineiramente, para hidratação e regeneração labial com pomada de dexpantenol, além de suspensão de um hidróxido de alumínio/magnésio e simeticona para as áreas com 
lesões mais extensas observadas intraoral, bem como o protocolo de higiene oral estabelecido pela equipe de terapia intensiva com o uso de digluconato de clorexidina $0,12 \%$.

Em 72 horas após a primeira aplicação de PBMT foi observado intensa formação de tecido de granulação em toda mucosa oral e diminuição do aspecto necrótico em língua, assim como a diminuição parcial do edema, facilitando o acesso do cirurgião dentista a cavidade oral, como pode ser observado nas figuras a seguir, lesões com aspecto mais cruento, tecido de granulação em língua e edema parcialmente diminuído o que facilita a abertura bucal, assim como observado na figura a seguir:

Figura 2: A: Segundo dia de aplicação da LLLT, úlceras sangrantes em mucosas labiais, língua com presença de tecido de granulação. B: Diminuição parcial do edema sublingual com acomodação de língua.

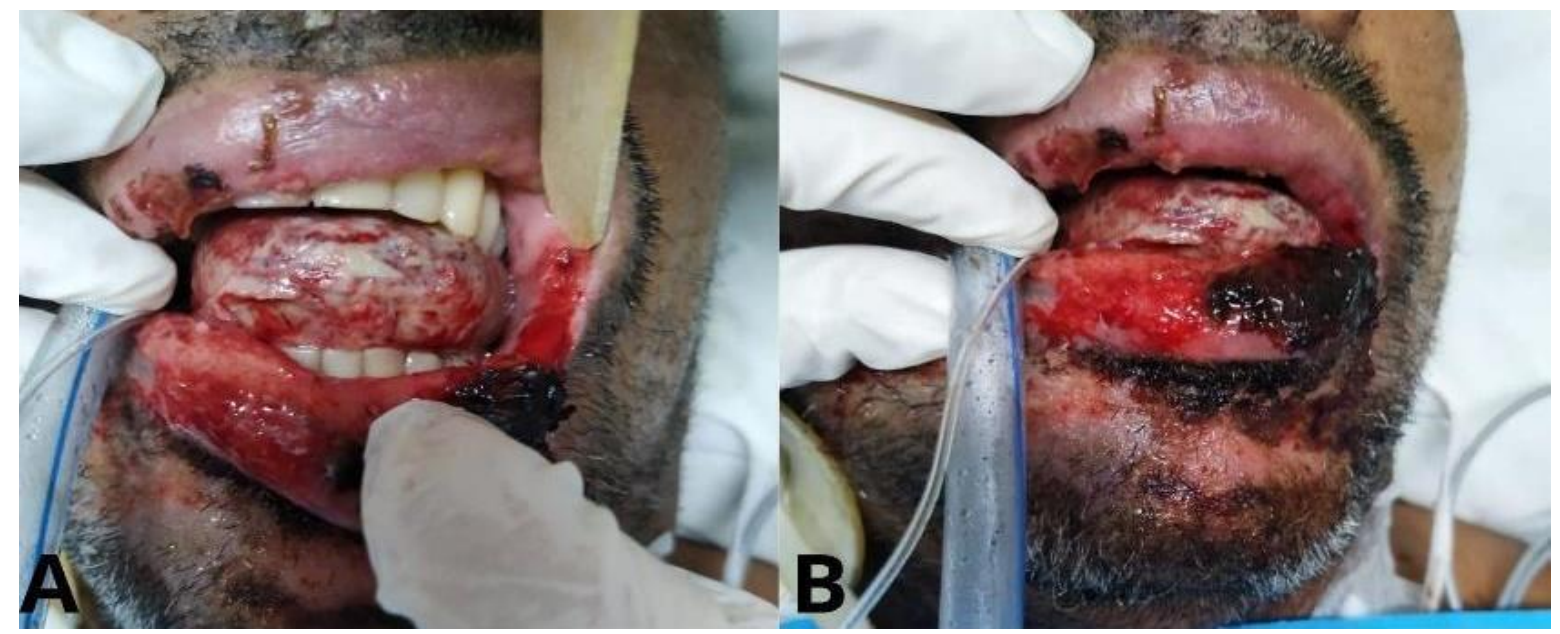

Fonte: Autores.

Em 9 dias consecutivos de aplicação de terapia com laser de baixa intensidade as feridas começaram a apresentar melhora significativa na cicatrização assim como nos dias que se seguiram, as lesões por queimadura química se encontravam em estágio avançado de cicatrização sendo que o paciente não referia dor espontânea, a seguir figura demonstrando cicatrização total: 
Figura 3: A: Após 9 dias de aplicação LLLT, lábios e mucosas labiais completamente cicatrizados, com presença de microstomia. Ápice lingual ainda com lesão ulcerada de pequena proporção não sangrante e não dolorosa. B: Lábios completamente cicatrizados após aplicações de LLLT, restando cicatrizes perilabiais.

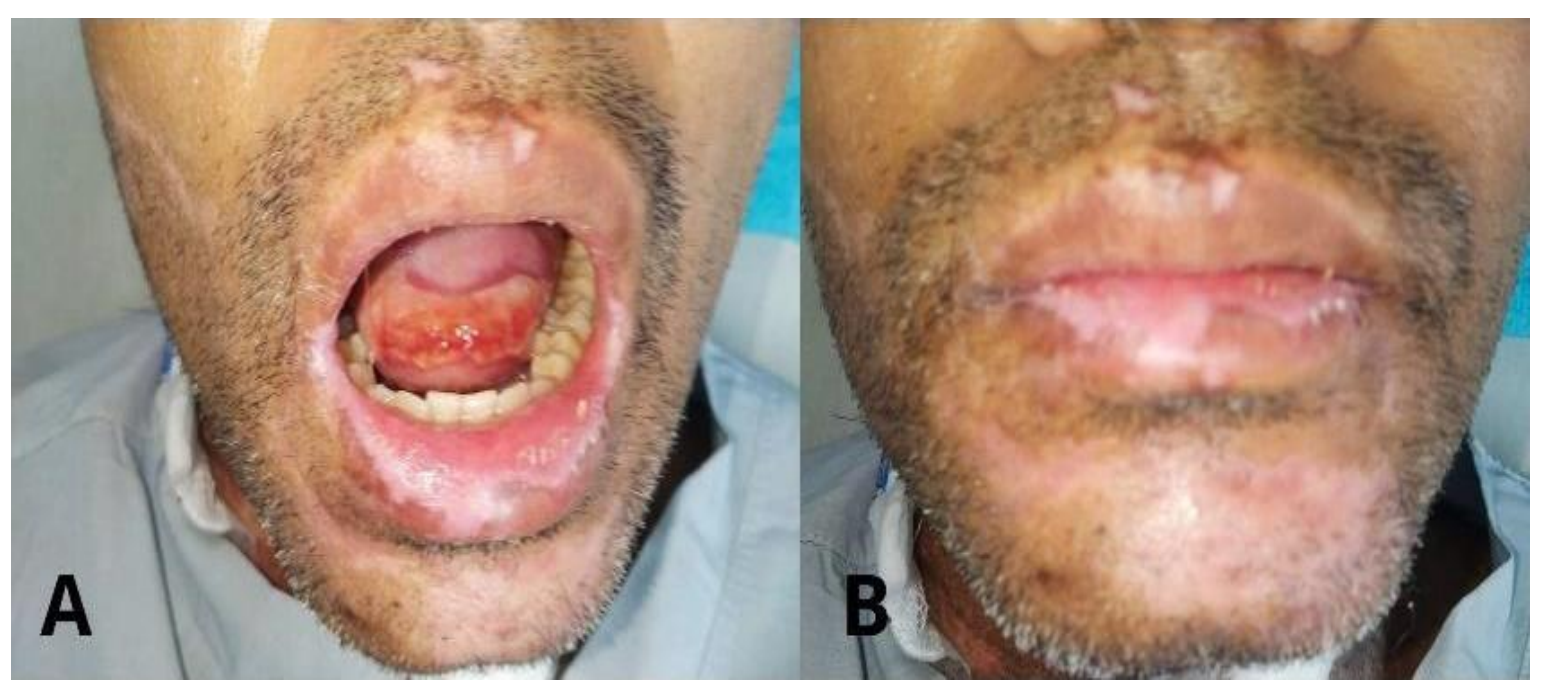

Fonte: Autores.

Mediante melhora expressiva da condição sistêmica do paciente, totalizados 27 dias de internação, sendo 15 dias de internação em Unidade de Terapia Intensiva, ele recebeu alta hospitalar, com orientações para a continuidade do acompanhamento pela via ambulatorial uma vez por semana no intuito de manutenção do cuidado especializado, integral e longitudinal junto a equipe multiprofissional responsável.

Em relação a situação da cavidade bucal, paciente seguiu com o uso de atropina para diminuição do padrão salivar, uma vez que o mesmo em decorrência do procedimento cirúrgico gástrico foi impossibilitado de realizar deglutição, além da observação de microstomia, sendo que o paciente ainda se encontra em acompanhamento semanal para os devidos cuidados de manutenção da saúde oral.

\section{Discussão}

A utilização da terapia fotodinâmica antimicrobiana (aPDT) foi considerada tendo em vista seu potencial de descontaminação local e prevenção de infecções, já a associação com laserterapia de baixa potência objetivou a modulação do quadro inflamatório instalado, melhoria do processo cicatricial para lesões multiloculares e sobretudo no alívio da sensação álgica, a fotobiomodulação estimula o processo de reparação das injúrias teciduais e reduz significativamente as dimensões do edema local, refletindo positivamente sobre redução da dor. A utilização do LLLT favorece a estimulação da produção de colágeno, diminui o exsudato inflamatório intensificando a revascularização de maneira a otimizar a epitelização das feridas (Alamoudi et al., 2020; Lemes et al., 2018; Noda et al., 2016).

Oportunamente Mosca et al. (2019) relataram a interação do tecido biológico com o estímulo luminoso apontando o alívio da dor e inflamação, modulação do sistema imunológico que aumenta a capacidade de cicatrização e regeneração dos tecidos, segundo Hamblin (2018) uma das explicações, e a mais estudada para tal fato se baseia na absorção de determinados comprimentos de onda e luz específicos do espectro vermelho $(580-700 \mathrm{~nm})$ dentro da cadeia respiratória da mitocôndria celular, ativando a via CCO (citocromo C oxidase) diminuindo o stress oxidativo celular.

Sendo constatado sob mesmo espectro de luz vermelha $(580$ - $700 \mathrm{~nm})$ a redução significativa na concentração de interleucinas pró inflamatórias bem como a diminuição da concentração de fator de necrose tumoral (TNF-a). A irradiação de 
feridas no comprimento de onda vermelho (580- 700nm) está relacionada a inibição de processos inflamatórios, angiogênese e estimulação da proliferação de fibroblastos em contrapartida a terapia com o laser em comprimento de onda infravermelho (980 - 808nm) não afeta significativamente os processos de cicatrização tecidual o que justifica a escolha do espectro vermelho para o presente caso (Taradaj et al., 2018).

A associação da aPDT, que inclui a mistura de um fotossensibilizador não tóxico e uma fonte de luz constituem proposta terapêutica eficaz contra a ampla gama de bactérias da cavidade oral além de cepas multirresistentes como Acinetobacter baumannii, Klebsiella pneumoniae e Pseudomonas aeruginosa (Javali et al., 2019) (Sabino et al., 2020).

Desta feita, a evolução clínica acompanhada nesta casuística evidenciou a aPDT e PBMT como agentes responsáveis por significativa melhora clínica da condição oral do paciente, especificamente em relação à remissão das lesões e do edema em aproximadamente nove dias. Cabendo a ressalva de que mesmo com a evolução favorável do quadro clínico da mucosa oral, ainda pudemos observar a exacerbação cicatricial da região perioral evoluindo para um quadro de microstomia e estenose cutânea da comissura labial, efeito adverso comumente observado em decorrência de queimaduras químicas, porém passível de intervenção cirúrgica corretiva posteriormente (Nunes \& Nemr, 2005).

A importante redução do edema obtida com a fotobiomodulação corrobora com a literatura consultada (Lemes, et al., 2018; Noda et al., 2016; Oliveira, 2020) justificando a ação do laser diretamente nas fases iniciais do processo de reparo, aumentando o metabolismo celular e a síntese de endorfinas com consequente redução da liberação de citocinas pró inflamatórias, do mesmo modo que aumenta o fluxo sanguíneo e a drenagem linfática associado ao uso da aPDT que é significativamente eficaz contra patógenos locais (Yang et al., 2020).

\section{Considerações Finais}

O presente exposto sugere que a terapia fotodinâmica antimicrobiana associada à laserterapia de fotobiomodulação de baixa potência pode ser adotadas como conduta viável na reabilitação de pacientes portadores de queimaduras por substâncias cáusticas em mucosa oral. Favorecendo o processo reparador de forma não invasiva, diminuindo a chance de contaminação, reduzindo edema, estimulando a formação de tecido reparador e sobretudo aliviando a dor do paciente. O conhecimento do comportamento dos tecidos bucais bem como da eficácia de propostas terapêuticas associadas, corrobora para a consolidação do Cirurgião Dentista devidamente habilitado, como profissional de atuação necessário no ambiente hospitalar.

Este artigo apresenta limitações intrínsecas a um relato de caso, logo novos trabalhos devem ser realizados para que seja alcançada e aumentada a confiabilidade estatística dos resultados obtidos.

\section{Referências}

Alamoudi, N., Nadhreen, A., Sabbagh, H., El Meligy, O., Al Tuwirqi, A., \& Elkhodary, H. (2020). Clinical and Radiographic Success of Low-Level Laser Therapy Compared with Formocresol Pulpotomy Treatment in Primary Molars. Pediatric dentistry, 42(5), 359-366.

Bittencourt, P. F. S.; \& Carvalho, S. D. (2018). Caustic accidents in childhood - a proposal for care in the acute and chronic phases of the accident. Revista médica de Minas Gerais, 28 (6).

Gilvetti, C.; Porter, S. R.; \& Fedele, S. (2010). Traumatic chemical oral ulceration: a case report and review of the literature. Brazilian Dental Journal, 208(7) 297-300.

Varkey P, Tan N. C., \& Chen, H. C. Corrosive injury of oral cavity-a rare presentation. 2006. Jounal Plast Reconstr Aesthet Surg, 59(10):1110-3. 10.1016/j.bjps.2006.03.069.

Hamblin M. R. (2018). Mechanisms and Mitochondrial Redox Signaling in Photobiomodulation. Photochemistry and photobiology, 94(2), 199-212.

Javali, M. A., AlQahtani, N. A., Ahmad, I., \& Ahmad, I. (2019) Antimicrobial photodynamic therapy (light source; methylene blue; titanium dioxide): Bactericidal effects analysis on oral plaque bacteria: An in vitro study. Nigerian journal of clinical practice, 22(12), 1654-1661. 
Research, Society and Development, v. 10, n. 15, e44101522340, 2021

Lemes, C., da Rosa, W., Sonego, C. L., Lemes, B. J., Moraes, R. R., \& da Silva, A. F. (2019). Does laser therapy improve the wound healing process after tooth extraction? A systematic review. Wound repair and regeneration: official publication of the Wound Healing Society [and] the European Tissue Repair Society, 27(1), 102-113.

Mosca, R. C., Ong, A. A., Albasha, O., Bass, K., \& Arany, P. (2019). Photobiomodulation Therapy for Wound Care: A Potent, Noninvasive, Photoceutical Approach. Advances in skin \& wound care, 32(4), 157-167.

Noda, M., Aoki, A., Mizutani, K., Lin, T., Komaki, M., Shibata, S., \& Izumi, Y. (2016). High-frequency pulsed low-level diode laser therapy accelerates wound healing of tooth extraction socket: An in vivo study. Lasers in surgery and medicine, 48(10), 955-964.

Nunes, J., \& Nemr, K. (20050. Queimaduras e as alterações miofuncionais e laríngeas. Revista CEFAC. 7. 466-472.

Oliveira, C. G., Freitas, M. F., de Sousa, M., Giorgi, R., \& Chacur, M. (2020). Photobiomodulation reduces nociception and edema in a CFA-induced muscle pain model: effects of LLLT and LEDT. Photochemical \& photobiological sciences: Official journal of the European Photochemistry Association and the European Society for Photobiology, 19(10), 1392-1401.

Pereira, P. A. (2018). Metodologia da pesquisa científica. UFSM.

Raffaele, R. M., Baldo, M. E., Queiroz, P. F. S., Tateno, R. Y., Palma L. F., \& Campos, L. Phototherapies in the management of an ulcerative lip lesion in a patient taking multiple medications for rheumatoid arthritis. Research, Society and Development.10(8), e50210817538.

Raffaele, R. M., De Oliveira, R. L., Baldo, M. E., Santana, G. U., \& Pacheco, J. A. (2020). Treatment of Low Level Laser Therapy in the Oral Manifestations of Steven Johnson Syndrome. International Journal of Emergency Medicine. 1(3):1-8.

Sabino, C. P., Wainwright, M., Ribeiro, M. S., Sellera, F. P., Dos Anjos, C., Baptista, M., \& Lincopan, N. (2020). Global priority multidrug-resistant pathogens do not resist photodynamic therapy. Journal of photochemistry and photobiology. B, Biology, $208,111893$.

Slaughter, R. J., Watts, M., Vale, J. A., Grieve, J. R., \& Schep, L. J. (2019). The clinical toxicology of sodium hypochlorite. Clinical toxicology (Philadelphia, Pa.), 57(5), 303-311.

Souza, M. T., Silva, M. D., Carvalho, R. (2021). Revisão Integrativa: o que é e como fazer. Einstein, 8 (1 Pt 1): $102-6$.

Taradaj, J., et al (2018). Effect of laser therapy on expression of angio- and fibrogenic factors, and cytokine concentrations during the healing process of human pressure ulcers. International Journal of Medical Sciences. 15. 1105-1112.

Rodríguez V., Briny O, Monge S. E., Montes T., Pedro, Ventura P., S., \& Guzmán C., E. (2016). Lesiones por cáusticos del tracto digestivo superior: características clínicas y endoscópicas. Revista de Gastroenterología del Perú, 36(2), 135-142.

Varkey, P., Tan, N. C., \& Chen, H. C. (2006). Corrosive injury of oral cavity--a rare presentation. Journal of plastic, reconstructive \& aesthetic surgery : JPRAS, 59(10), 1110-1113.

Yang, T., Tan, Y., Zhang, W., Yang, W., Luo, J., Chen, L., Liu, H., Yang, G., \& Lei, X. (2020). Effects of ALA-PDT on the Healing of Mouse Skin Wounds Infected With Pseudomonas aeruginosa and Its Related Mechanisms. Frontiers in cell and developmental biology, 8 , 585132. https://doi.org/10.3389/fcell.2020.585132. 